\title{
Encoding of emotional memories depends on amygdala and hippocampus and their interactions
}

\author{
Mark P Richardson ${ }^{1}$, Bryan A Strange ${ }^{2} \&$ Raymond J Dolan ${ }^{2}$ \\ We have studied patients with variable degrees of left hippocampal and amygdala pathology who performed a verbal encoding \\ task during functional magnetic resonance imaging (fMRI) to assess the impact of pathology on emotional-memory performance \\ and encoding-evoked activity. The severity of left hippocampal pathology predicted memory performance for neutral and \\ emotional items alike, whereas the severity of amygdala pathology predicted memory performance for emotional items alone. \\ Encoding-related hippocampal activity for successfully remembered emotional items correlated with the degree of left amygdala \\ pathology. Conversely, amygdala-evoked activity with respect to subsequently remembered emotional items correlated with the \\ degree of left hippocampal pathology. Our data indicate a reciprocal dependence between amygdala and hippocampus during the \\ encoding of emotional memories.
}

Experiments in humans ${ }^{1}$ and animals ${ }^{2}$ indicate an advantage in memory for emotional as compared with neutral items. Many lines of evidence point to the amygdala as a locus of enhanced activity in response to emotional stimuli ${ }^{3}$, particularly for those of a fearful or aversive nature. Functional imaging experiments in normal subjects ${ }^{4}$ and behavioral studies in patients with amygdala lesions ${ }^{5,6}$ confirm the role of the amygdala in the perception and processing of fear. For example, enhanced amygdala activity occurs in response to emotionally aversive 'oddball' stimuli that are presented in a neutral context ${ }^{7}$. In normal subjects, amygdala activity for emotional stimuli correlates with subsequent memory of those items ${ }^{8}$. Evidence from animal data indicates that memory enhancement for emotional items may reflect a modulatory influence of the amygdala on the hippocampus ${ }^{2,9}$.

The hippocampus is critical for episodic memory ${ }^{10}$, with left and right hippocampal injury leading to impairment in verbal and nonverbal memory function, respectively ${ }^{11,12}$. Although hippocampal activity correlates with subsequent memory for neutral items ${ }^{13}$, neuropsychological and functional imaging studies have yet to provide evidence for an amygdala-mediated modulation of hippocampal function during the encoding of emotional items. Consequently, we have studied patients with varying degrees of left medial temporal pathology to assess amygdala-hippocampal cooperativity during emotional-memory encoding.

The modern clinical investigation of patients with temporal lobe epilepsy involves acquisition of structural MRI of the brain, with subsequent measurement of medial temporal structures using highresolution T1-weighted imaging ${ }^{14}$. Additionally, the integrity of residual brain tissues is measured using a dual-echo T2-weighted image, allowing measurement of T2 relaxation on a voxel-by-voxel basis throughout the brain, known as a T2 map ${ }^{15,16}$. Combining these techniques allows identification of a common pathology, hippocampal sclerosis, with high sensitivity and specificity ${ }^{15,17}$. The hallmark of hippocampal sclerosis is reduced hippocampal volume and increased hippocampal T2 signal, both of which vary in severity in a given patient. This pathology is highly variable in its extent, being either unilateral or bilateral and with or without amygdala involvement ${ }^{18}$. Notably, the severity of sclerosis in one region does not correlate with the severity in other regions ${ }^{19}$. Patients with medial temporal sclerosis typically have mild material-specific memory impairment related to the side of pathology, although performance is often within the normal range $e^{20}$. Although pathology may be accurately detected by measurement of brain structure in candidate regions using MRI, the technique of voxel-based morphometry $(\mathrm{VBM})^{21}$ allows detection of the precise location of a structural brain abnormality without the need for an a priori anatomical hypothesis. VBM requires conventional T1weighted images, which are transformed to a standard brain volume and are segmented to produce images of gray-matter density (GMD).

To examine amygdala-hippocampal interactions during emotional-memory encoding, we studied verbal encoding of neutral and emotional stimuli using structural MRI and event-related functional $\mathrm{fMRI}^{22}$. We tested 16 right-handed patients, all of whom had left hippocampal sclerosis of variable severity, with or without additional left amygdala sclerosis. All the patients had normal right hippocampal measurements. We also studied 12 normal control subjects. During fMRI, the subjects viewed 288 single words on a screen, of which $12.5 \%$ were emotionally aversive ('cancer', 'rape', 'terrorist'), while performing a deep-encoding task ${ }^{23}$. Subsequently, all subjects performed an unexpected recognition test, indicating whether a particular word was definitely remembered ('remember' response; R), familiar but not definitely remembered ('know' response; K), or not seen previously ('new'; N ) ${ }^{24--}$. The stimuli from the scanned encoding session were subsequently conditionalized according to these

${ }^{1}$ Department of Clinical and Experimental Epilepsy, Institute of Neurology, Queen Square London WC1N 3BG, UK. ${ }^{2}$ Wellcome Department of Imaging Neuroscience, Institute of Neurology, Queen Square London WC1N 3BG, UK. Correspondence should be addressed to M.P.R. (m.richardson@ion.ucl.ac.uk). 


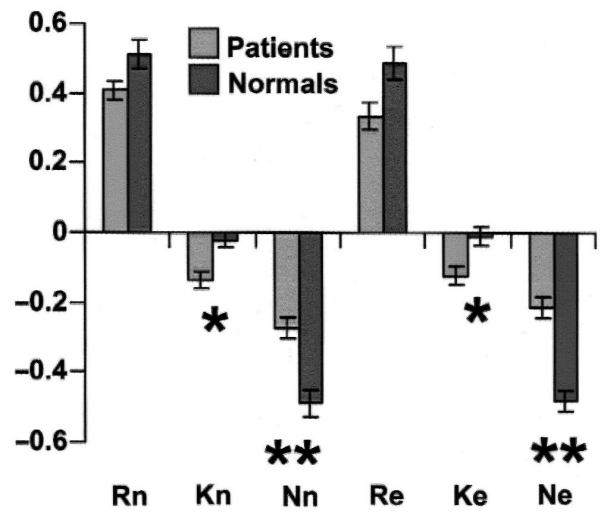

responses such that fMRI scanning data were analyzed to index encoding activity specific to each of these subsequent memory types.

Our analyses involved two approaches. To determine a general measure of the effects of medial temporal damage on memory, we first examined the relationship between recognition accuracy and structural imaging indices (hippocampal volumes, voxel-by-voxel GMD, voxel-by-voxel T2 signal) by correlating severity of pathology with recognition accuracy. This showed that the severity of left hippocampal pathology predicted memory performance for both neutral and emotional items, whereas the severity of amygdala pathology predicted memory for emotional items alone. To examine amygdalahippocampal co-dependence during emotional-memory encoding, we then ascertained the relationship between structural imaging indices and the magnitude of encoding-related activity at each voxel of
Figure 1 Recognition accuracy for each response type in patients and normal controls. A recognition accuracy score of +1 indicates perfect identification of stimuli seen at encoding; -1 indicates perfect recognition of foils. Rn, Kn, Nn: 'remember', 'know' and 'new' responses for neutral items; $\mathrm{Re}, \mathrm{Ke}, \mathrm{Ne}$ : 'remember', 'know' and 'new' responses for emotional items. ${ }^{*} P<0.01$ and ${ }^{* *} P<0.001$ as compared between patients and normal subjects. There is no interaction between recognition accuracy, emotionality and subject group (repeated measures ANOVA, $F_{1,26}=1.38, P=0.25$ ).

the fMRI data. We show that encoding-related hippocampal activity for successfully remembered emotional items correlates with the degree of left amygdala pathology. Conversely, amygdala-evoked activity to subsequently remembered emotional items correlated with the degree of left hippocampal pathology.

\section{RESULTS}

\section{Behavioral data}

Recognition accuracy (Fig. 1) was defined as the rate of true positives minus the rate of false positives. Patients made fewer $\mathrm{N}$ responses (mean: 28.8 for patients, 54.3 for controls; $P=0.026$, two-tailed unpaired $t$-test) but the same number of $\mathrm{K}$ (mean: 40.4 for patients, 42.8 for controls) and R (mean: 162.8 for patients, 164.4 for controls) responses as compared with controls. On the basis of these data, we chose the contrast of $\mathrm{R}$ minus $\mathrm{K}$ to represent subsequent memory effect in the fMRI data. Our behavioral data did not show enhancement of recognition for emotional items in patient or control groups, a finding in agreement with another study of emotional item recognition $^{25}$ in which the recognition test was undertaken after a short interval, as in our study. In a previous experiment, using the same
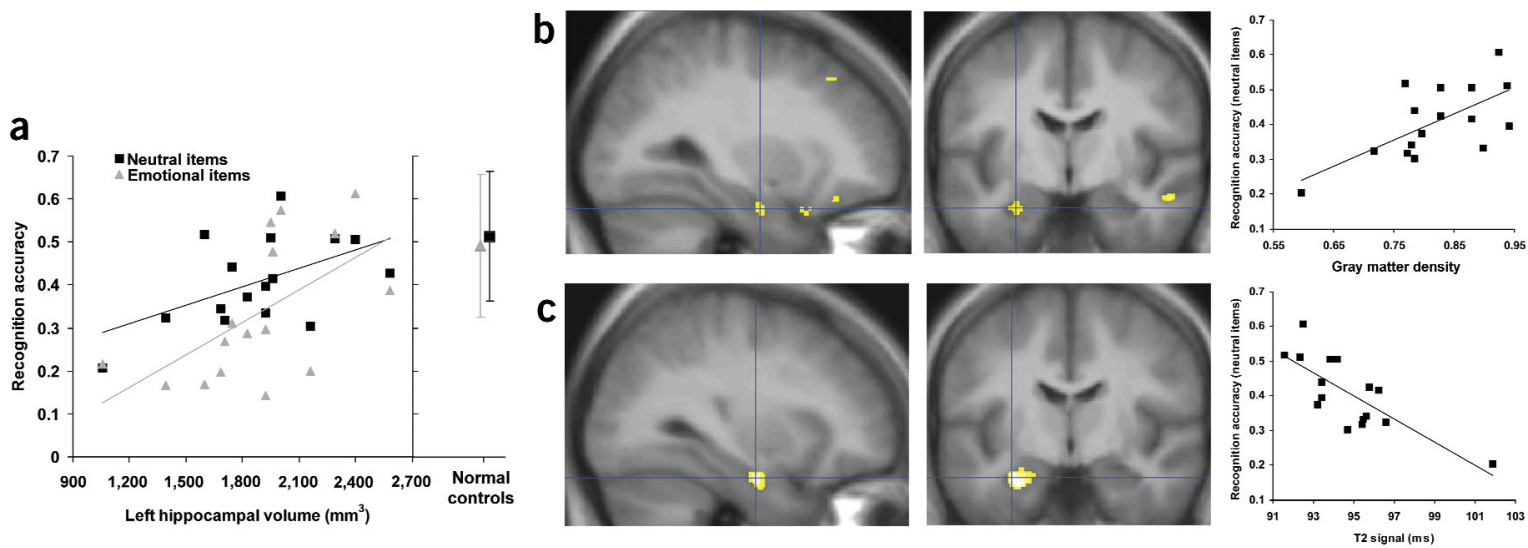

Figure 2 Structure-behavior relationships. (a) Recognition accuracy for R responses to neutral items and emotional items is significantly correlated with left hippocampal volume, as corrected for total intracranial volume $\left(\mathrm{mm}^{3}\right)$. For comparison with the patient data, the normal control recognition accuracy data (mean \pm 1 s.d.) is illustrated alongside the patient data. (b) Statistical parametric map (SPM) overlaid on sagittal and coronal sections of the averaged T1 image of all 16 patients studied (threshold here and all subsequent figures, $P<0.01$ ). Units for GMD are arbitrary. (c) SPM showing a significant negative correlation in left hippocampus between $\mathrm{T} 2$ signal and recognition accuracy for $\mathrm{R}$ responses to neutral items. (d) Left amygdala ( $-296-15, Z=3.05)$ shows a significant positive correlation between GMD and recognition accuracy for $\mathrm{R}$ responses to emotional items. (e) SPM showing a significant interaction between GMD, recognition accuracy for $R$ responses and emotionality in left amygdala $(-24-3-15, Z=2.48)$.
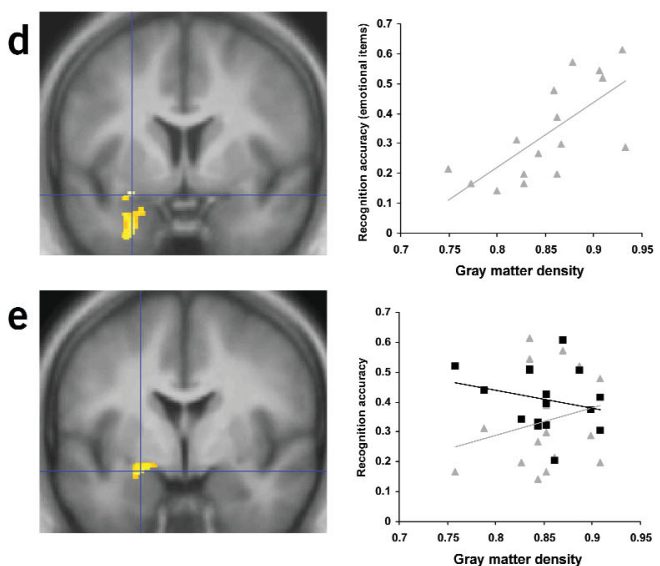


\section{ARTICLES}

Table 1 Summary of effects reported for structural and functional imaging data

\begin{tabular}{|c|c|c|c|c|c|c|}
\hline Effect examined & $\begin{array}{l}\text { Result illustrated } \\
\text { in figure }\end{array}$ & $Z$ score & $\begin{array}{l}\text { Uncorrected } \\
P \text { value }\end{array}$ & $\begin{array}{l}\text { Corrected } P \text { value } \\
\text { (small volume used) }\end{array}$ & $\begin{array}{l}\text { Coordinate }(x y z) \\
\text { in MNI space }\end{array}$ & Anatomical region \\
\hline \multicolumn{7}{|l|}{ Structure-behavior relationships } \\
\hline $\begin{array}{l}\text { GMD versus recognition accuracy } \\
\text { for } \mathrm{Rn}\end{array}$ & Figure $2 b$ & 2.72 & 0.003 & NS (ha) & $-29-8-24$ & Left hippocampus \\
\hline $\begin{array}{l}\text { T2 signal versus recognition accuracy } \\
\text { for } \mathrm{Rn}\end{array}$ & Figure $2 c$ & 3.51 & $<0.001$ & 0.038 (ha) & $-32-9-22$ & Left hippocampus \\
\hline $\begin{array}{l}\text { GMD versus recognition accuracy } \\
\text { for } R e\end{array}$ & Figure 2d & 3.05 & 0.001 & 0.017 (a) & $-296-15$ & Left amygdala \\
\hline Interaction between & Figure $2 \mathrm{e}$ & 2.48 & 0.007 & 0.06 (a) & $-24-3-15$ & Left amygdala \\
\hline
\end{tabular}

GMD, recognition accuracy

for R and emotionality

Structure-function relationships

Left hippocampal volume versus successful encoding-related

activation for neutral items

Left hippocampal volume versus successful encoding-related

$3.41<0.001$

$26-12-12$

Right hippocampus

activation for neutral items

Left hippocampal T2 signal versus

Figure 3c

3.60

$<0.001$

0.023 (ha)

$26-18-10$

Right hippocampus

successful encoding-related

activation for neutral items

Amygdala-hippocampal co-dependence during successful encoding of emotional items

Interaction between

Figure 4a

2.56

0.005

$0.016(h)$

$-32-18-20$

Left hippocampus

left amygdala T2,

successful encoding-activation

and emotionality

Interaction between

left amygdala T2,

Figure 4b

2.65

0.004

NS (ha)

$24-12-12$

Right hippocampus

successful encoding-activation

and emotionality

Interaction between

left hippocampal volume,

Figure 4c

3.05

0.001

0.013 (a)

$-20-6-12$

Left amygdala

successful encoding-activation

and emotionality

Interaction between

Figure 4d

2.66

0.004

$0.033(a)$

$30-8-14$

Right amygdala

successful encoding-activation

and emotionality

NS, nonsignificant; ha, small volume comprising hippocampus and amygdala; h, small volume in left hippocampus; a, small volume in amygdala.

items as were used in this experiment, an enhancement in free recall for emotional as compared with neutral items was observed ${ }^{7}$. The similarity in the behavior observed for neutral and emotional items in this study allows us to conclude that any differences in the imaging data that correlate with emotionality must be a consequence of emotionality and not a consequence of a behavioral difference. We note that normal control subjects, who provided behavioral comparison, showed a main effect of encoding, as determined with a comparison of $\mathrm{R}$ minus $\mathrm{K}$, in left hippocampus $(-32,-18,-18)^{26}$.

\section{Structure-behavior relationships}

We first examined the data for an effect of medial temporal lobe (MTL) pathology on encoding success. The left and right total hip- pocampal volumes of all patients were measured from T1-weighted MRI according to a standard protocol ${ }^{27,28}$. Recognition accuracy ( R responses) for neutral items was correlated with left hippocampal volume (Fig. $2 \mathrm{a} ; R=0.521, P=0.038$ ), as was recognition accuracy for emotional items $(R=0.591, P=0.016$; no significant interaction). Thus, as the left hippocampal volume decreased, so did the recognition accuracy.

To improve anatomical specificity of our findings within the MTL, we used $\mathrm{VBM}^{21}$. Regression of recognition accuracy ( $\mathrm{R}$ responses for neutral items) against GMD at all voxels identified a single MTL region that showed a significant correlation with recognition accuracy for neutral items in anterior left hippocampus (29 -8 -24, $Z=2.72$; Fig. 2b; Table 1). Subjects with less gray matter in the ante- 

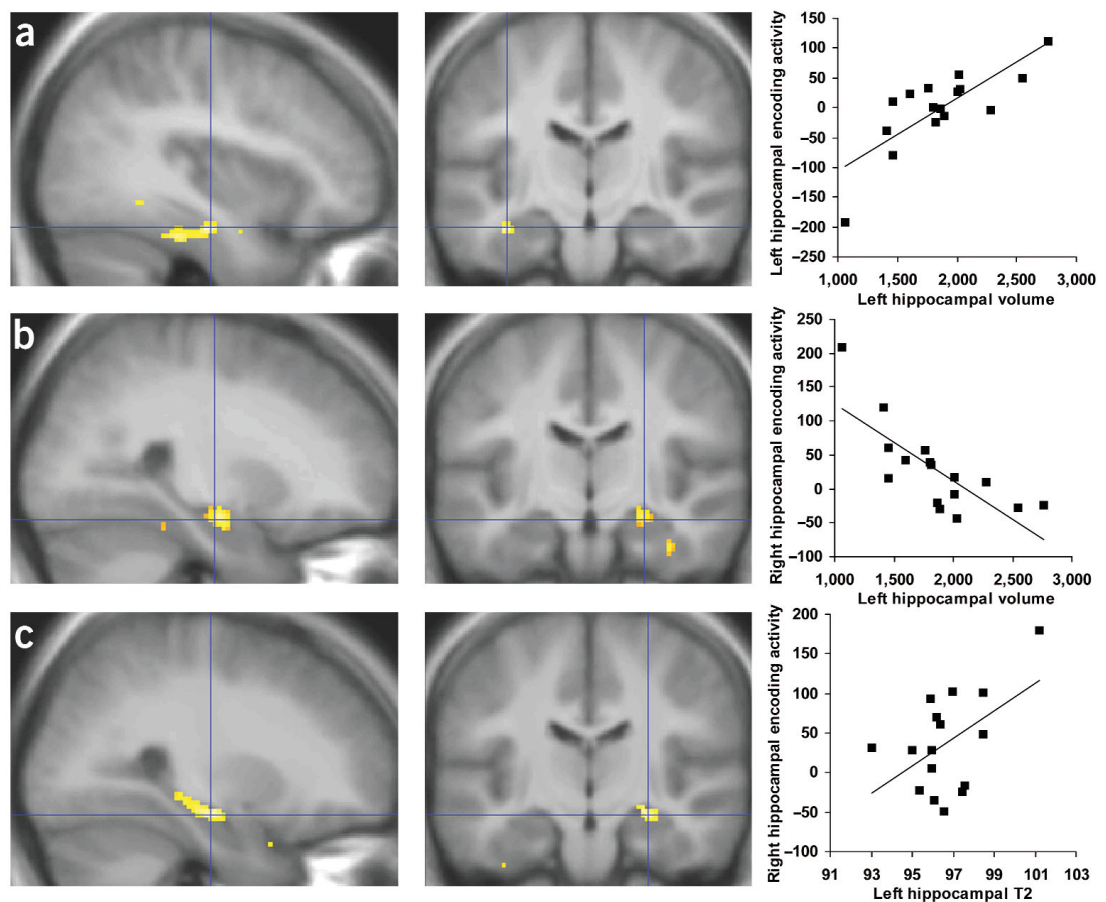

Figure 3 Structure-function relationships. (a) The SPM illustrates a significant positive correlation in left hippocampus $(-38-14-18, Z=3.62)$ between total left hippocampal volume $\left(\mathrm{mm}^{3}\right)$ and successful encoding-related activation ( $R$ minus $\mathrm{K}$ ) for neutral items. Encoding-related activity units are arbitrary. (b) SPM showing a significant negative correlation between total left hippocampal volume and successful encoding-related activation ( $R$ minus $K$ ) for neutral items in right hippocampus (26 $-12-12, Z=3.41)$. There is a strong inverse correlation between the activity in left hippocampus, as shown in a, and right hippocampus $(R=0.75, P=0.0009)$. (c) Right hippocampus $(26-18-10, Z=3.60)$ shows a significant positive correlation between left hippocampal T2 signal and successful encoding-related activation (R minus $\mathrm{K}$ ) for neutral items, with total left hippocampal volume as a covariate of no interest.

rior left hippocampus had worse recognition. In an analogous manner, T2 maps, which provide an alternative index of pathology, were entered into a regression against recognition accuracy ( $\mathrm{R}$ responses for neutral items). The same left hippocampal region $(-32-9-22$, $Z=3.51$ ) showed a negative correlation between $\mathrm{T} 2$ signal and recognition accuracy (Fig. 2c). Strikingly, the only voxels in the entire brain to show significant correlations for both comparisons were in this left hippocampal region (see Supplementary Fig. 1 online), resulting in a highly anatomically specific prediction of recognition accuracy.

Also using VBM, we showed that less gray matter in the antero-lateral and superior margins of the left amygdala correlated with poorer recognition accuracy for emotional items (Fig. 2d). At a lower level of significance, we observed a three-way interaction in the left amygdala between recognition accuracy, emotionality and GMD (Fig. 2e). This reflects a significantly greater cost with loss of gray matter in the left amygdala with respect to recognition accuracy of emotional items as compared with that of neutral items. There were no significant correlations between recognition of emotional items and T2 signal.

It is of note that there was no correlation between measures of pathology in the left amygdala and left hippocampus. Among the peak voxels in the VBM data, there was no correlation between left hippocampal GMD and left amygdala GMD, using either the amygdala region showing strongest correlation with emotional-item recognition accuracy $(R=0.15$; Fig. $2 \mathrm{~d})$ or the amygdala region showing an interaction between GMD, recognition accuracy and emotionality $(R=$ 0.093; Fig. 2e). Likewise, there was also no correlation between left hip- pocampal T2 and left amygdala T2 $(R=0.21)$. As expected, left hippocampal volume and left hippocampal T2 are correlated $(R=0.47)$. Thus, the lack of correlation between the severity of left amygdala and left hippocampal pathology allows us to examine hippocampal and amygdala effects independently.

\section{Structure-function relationships}

Having confirmed an effect of MTL pathology on encoding success, we next tested for a relationship between site and severity of pathology and site and magnitude of encoding-related neuronal activity (measured with $\mathrm{fMRI}$ ) that was associated with a subsequent memory effect. An image of encoding activity ( $\mathrm{R}$ minus $\mathrm{K}$ ) for successfully recognized neutral items for each subject was entered into a regression against total left hippocampal volume (Fig. 3a). More severe left hippocampal pathology correlated with less encoding-related activity for subsequently remembered items in left hippocampus. Conversely, more severe left hippocampal pathology correlated with enhanced encoding-related activity in right hippocampus (Figs. 3b and 5). Thus, in subjects with smaller left hippocampi, encoding-related neuronal activity shows an incremental shift from left to right hippocampus. Similarly, mean T2 signal in the entire residual left hippocampus (partialing out the effect of overall volume loss) showed that this index of pathology also predicts successful-encoding activity for neutral items and is shifted to right hippocampus (Figs. 3c and 5). Thus, in subjects with more abnormal left hippocampal tissue (higher T2 signal), there is more encoding-related neuronal activity in the right hippocampus for items that are subsequently remembered.

We next looked for hippocampal-amygdala codependence during encoding of emotional items. We based these experiments on the findings we had already demonstrated in this dataset, specifically that successful encoding of emotional items is affected by amygdala pathology and that hippocampal pathology predicts a shift of lateralization of encoding activity for neutral items. Animal data indicate that an amygdala-dependent enhancement of hippocampal responses may occur during emotional-stimulus encoding ${ }^{2,9}$. Thus, we predicted that increasing amygdala pathology would reduce hippocampal responses during successful encoding of emotional stimuli. We tested for a three-way interaction between left amygdala T2 and successful-encoding activity ( $\mathrm{R}$ minus $\mathrm{K}$ ) for emotional versus neutral items (with left hippocampal volume included as a covariate of no interest). A significant interaction was observed in left anterior hippocampus (Fig. 4a), indicating that increasing severity of left amygdala damage reduces successful encoding-evoked activation of emotional stimuli in hippocampus, which supports our hypothesis. The reverse correlation showed an interaction in right hippocampus (Figs. $4 \mathrm{~b}$ and 5), indicating that right hippocampal encoding-evoked activation for emotional versus neutral stimuli is enhanced with increasing severity of left amygdala pathology. Therefore, left amygdala damage results in a left-right shift in hippocampal responses 
during successful encoding of emotional words. Taking the activity at these left and right hippocampal peaks for each subject, we tested the hypothesis that left amygdala T2 determines the distribution of successful encoding-activity for emotional words between left and right hippocampus. Repeated measures analysis of variance (ANOVA) was used, with 'side' (left or right hippocampus) as the within-subjects factor and left amygdala T2 as the covariate. This analysis showed a significant interaction between the side of encoding activity and left amygdala $\mathrm{T} 2\left(F_{1,14}=6.69, P=0.02\right)$.

The proposal that ipsilateral hippocampus and amygdala are codependent during the encoding of emotional items predicts a reciprocal modulation of amygdala activity by hippocampus for successful encoding of emotional items. To assess this effect, we tested for a three-way interaction in the left amygdala between successful encoding-activity, left hippocampal volume and emotionality. A significant interaction expressed in left amygdala (Fig. 4c) indicates that more-severe hippocampal pathology results in decreased left amygdala activity for successful encoding of emotional as compared with neutral items. Note that this activation is in close approximation to the region showing an interaction between GMD, recognition accuracy and emotionality (Fig. 2e). The reverse correlation showed an interaction in right amygdala (Fig. 4d), indicating enhanced right amygdala encoding-evoked activation for emotional versus neutral stimuli with increasing severity of left hippocampal pathology. Therefore, left hippocampal damage results in a left-right shift in hippocampal and amygdala responses during successful encoding of neutral and emotional words, respectively. Taking the activity at these left and right amygdala peaks for each subject, we tested the hypothesis that left hippocampal volume determines the distribution of successful encoding-activity for emotional words between left and right amygdala. A repeated-measures ANOVA was used, with 'side' (left or right amygdala) as the within-subjects factor and left hippocampal volume as the covariate. This model showed a significant interaction between the side of encoding activity and left hippocampal volume $\left(F_{1,14}=27.31, P<0.0001\right)$.

\section{DISCUSSION}

Our analyses correlating pathology with behavior and correlating pathology with site of encoding activity provided a basis for our subsequent tests of amygdala-hippocampal codependence. We have shown that the severity of anterior left hippocampal pathology predicted subsequent recognition memory performance for both neutral and emotional items. A correlation between measures of verbal memory and left hippocampal volume has previously been shown ${ }^{29-31}$; we have extended these findings to show greater anatomical specificity using VBM. Volume loss and an increase in T2 signal reflect different aspects of hippocampal pathology: hippocampal volume loss is correlated with neuronal loss in the dentate hilus and CA1 regions of the hippocampus; elevation of T2 signal correlates with increased glial cell density, particularly in the dentate hilus ${ }^{27,32}$. In an analogous manner, the severity of left amygdala pathology predicted subsequent recognition memory performance for emotional items more than it did for neutral items. Although patients with isolated amygdala damage are known to show a specific impairment in recall of emotional stimuli ${ }^{6}$, a correlation between the severity of pathology and the severity of the deficit has not previously been demonstrated.

The severity of left hippocampal pathology also predicted the magnitude of encoding-related neuronal activity for neutral items in the left and right hippocampus: less-severe left hippocampal pathology predicted more activity in the left hippocampus and less in the right hippocampus; more severe pathology predicted the converse.
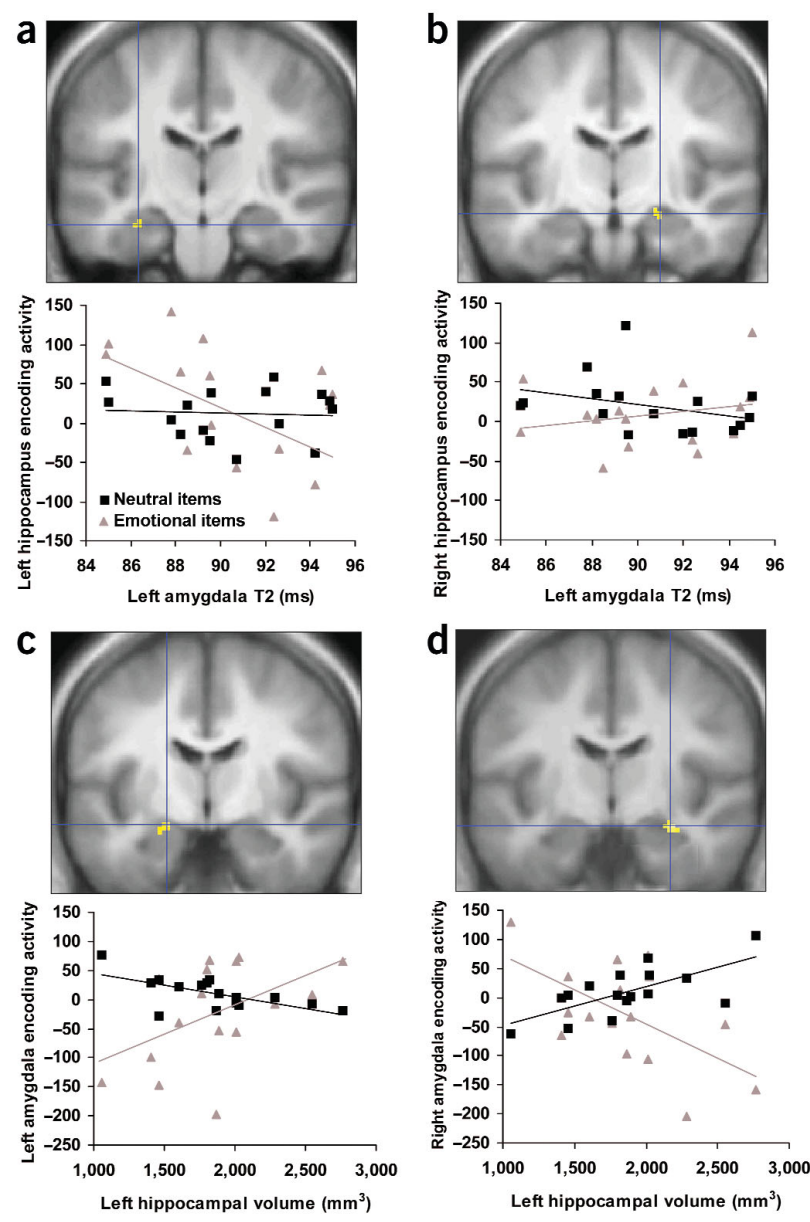

Figure 4 Amygdala-hippocampal reciprocal dependence during successful encoding of emotional words. (a) The SPM demonstrates a three-way interaction in left hippocampus $(-32-18-20, Z=2.56)$ between left amygdala T2 and successful-encoding activity ( $R$ minus $K$ ) for emotional versus neutral items (with left hippocampal volume included as a covariate of no interest). The graph shows the correlation between left amygdala T2 and successful encoding-related left hippocampal activation for emotional and neutral items. (b) The SPM demonstrates the other side of the three-way interaction between left amygdala T2 and successful-encoding activity ( $R$ minus K) for emotional versus neutral items in right hippocampus (24 -12 $-12, Z=2.65$ ). (c) SPM showing a three-way interaction between left hippocampal volume, successful encoding-related activation ( $R$ minus $K$ ) and emotionality (with left amygdala T2 as a covariate of no interest) in left amygdala $(-20-6-12, Z=3.05)$. The response estimates for successful encoding-related activity in left amygdala for emotional and neutral words are plotted against left hippocampal volume. (d) SPM demonstrating the other side of the three-way interaction between left hippocampal volume, successful encoding-related activation ( $\mathrm{R}$ minus $\mathrm{K}$ ) and emotionality (with left and right amygdala T2 as a covariates of no interest) in right amygdala $(30-8-14, Z=2.66)$.

Although hippocampal activity during memory tasks has been examined in patients with left hippocampal sclerosis using fMRI ${ }^{33-35}$, these studies did not demonstrate anterior hippocampal activity. Left hippocampal pathology was associated with a functional reorganization of both neutral and emotional verbal encoding to homologous regions of the right hippocampus and amygdala, respectively. As this study is of patients with lesions but good performance, we suggest that performance is preserved by neuronal reorganization of encoding-related processes to the right hippocampus and amygdala. 
a

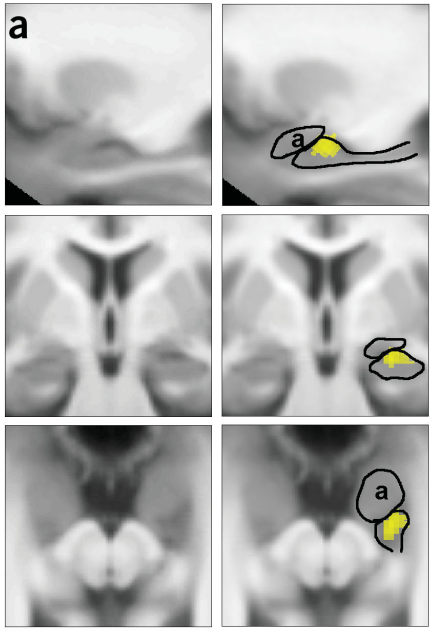

b

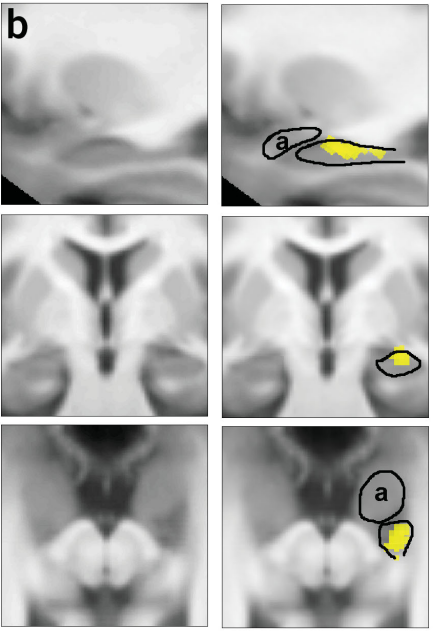

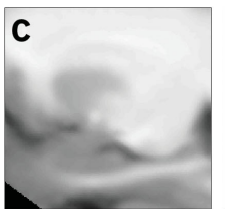
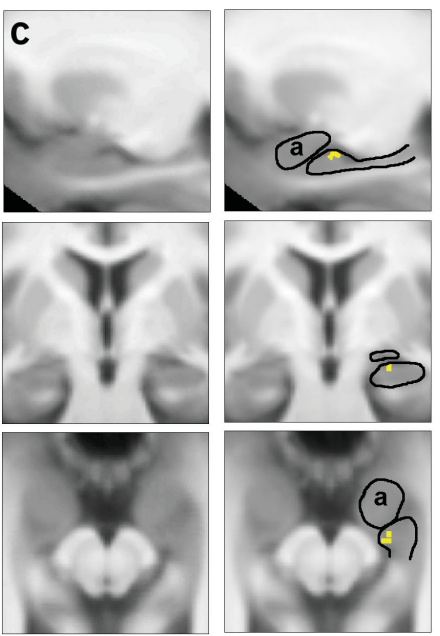

Figure 5 Anatomical specificity of hippocampal effects. The significant regions illustrated previously are shown here using a higher zoom; the images have been oriented so that the long axis of the hippocampus is parallel to the transverse sections. As in all of the figures, the T1-weighted MRI used to display the SPM data is the average T1-weighted MRI of the 16 patients; subtle left hippocampal volume loss is apparent (left is on the left). Each pair of columns of three images illustrates one significant region: top row, sagittal; middle row, coronal; lower row, transverse sections. The left-hand column of each pair shows the anatomy; the right-hand column shows superimposed on this the significant region and the approximate outlines of the hippocampus and amygdala. a, amygdala. (a) Hippocampal region from Figure 3b. (b) Hippocampal region from Figure 3c. (c) Hippocampal region from Figure 4b.

In a further demonstration of the anatomical specificity of our findings, we show that the distribution of encoding-related activity between left and right hippocampus predicts recognition accuracy for neutral items (Supplementary Note online). Likewise, the distribution of encoding-related activity between left and right amygdala predicts recognition accuracy for emotional items.

Functional imaging studies show that amygdala activity correlates with subsequent memory for emotional material in normal subjects ${ }^{4,7,8}$. The influence of the amygdala on the efficiency of encoding, however, is believed to be expressed through its effects on other structures, such as the hippocampus. Thus, our principal aim was to examine interactions between hippocampus and amygdala during encoding of emotional words. Left amygdala pathology as assessed by T2 measurements predicted reduced activity in the left hippocampus for emotional as compared with neutral items that were subsequently remembered. It is noteworthy that this is exactly the same hippocampal region where we previously demonstrated a main effect of subsequent memory in the normal subjects ${ }^{26}$. This observation supports the idea that the effects of amygdala activation on encoding are expressed in modulation of hippocampal activity in regions involved in verbal encoding operations 9 . Conversely, the severity of left hippocampal pathology predicts the magnitude of encoding-related neuronal activity for emotional items in the left and right amygdala: more-severe left hippocampal volume loss predicts less activity in left amygdala and more in right amygdala, whereas less-severe left hippocampal volume loss predicts the converse. Both findings indicate a mutual dependence between hippocampus and amygdala during encoding of emotional verbal material. Such 'cross-talk' between hippocampus and amygdala may permit the strong consolidation of an emotional memory ${ }^{36}$.

The modulation of hippocampal encoding-evoked activation by amygdala damage was observed in anterior hippocampus ( $y$ coordinate, -18 ). Anatomical studies in animals demonstrate that the anterior hippocampus is strongly reciprocally connected with the amygdala ${ }^{37}$, showing stronger connectivity than the posterior hippocampus. Both ventral (anterior) $\mathrm{CA}^{38}$ and anterior subiculum ${ }^{39}$ project to the amygdala. The reciprocal projection from amygdala to
CA1 terminates preferentially in the anterior third of this subfield. Amygdala projections to entorhinal cortex terminate primarily in medial entorhinal cortex, which projects to anterior dentate gyrus ${ }^{40}$. We suggest that this strong, reciprocal anatomical connectivity between anterior hippocampus and amygdala underlies their cooperativity during encoding of emotional stimuli.

In addition to anatomical evidence supporting a reciprocity between hippocampus and amygdala that subserves emotional memory, there is good physiological evidence for such interdependence. Tetanic stimulation of hippocampal efferents in the ventral anterior bundle of the rat induced long-term potentiation in the basolateral amygdala through a monosynaptic pathway ${ }^{41}$, indicating a possible substrate for hippocampal modulation of the amygdala. Furthermore, synchronization of amygdala and hippocampal theta rhythm during the expression of fear conditioning, indicates that interdependence is not only possible but crucial for behavioral manifestation of emotional memory ${ }^{42}$. To our knowledge, our data are the first demonstration that human amygdala activity is susceptible to modulation by the hippocampus.

The consistency of our findings provides compelling support for reciprocal interactions between left hippocampus and left amygdala in encoding of emotional words. The results show that both an intact left hippocampus and an intact left amygdala are required for optimal activity in the left hippocampus and optimal encoding of emotional items. The findings indicate that the hippocampus is a critical site for processes related to neuronal encoding, with reciprocal modulatory influences between the left amygdala and hippocampus being necessary for effective encoding of emotional material.

\section{METHODS}

Subjects. We studied 12 right-handed normal volunteers and 16 right-handed patients recruited from the National Hospital for Neurology and Neurosurgery, London, UK. All lived in southern England and were first-language English speakers. All patients had active temporal lobe epilepsy; age range was 18-54 years (median, 27); seven patients and eight normal subjects were male; all patients were on antiepileptic medication. All patients had undergone neuropsychometry and had a verbal IQ $>80$ (range, 84-121). Approval for this study was obtained from the Joint Research Ethics Committee of the Institute 
of Neurology and the National Hospital for Neurology and Neurosurgery, London, UK. All subjects gave informed consent.

Structural imaging. Structural MRI of the patients was carried out at 1.5T (Horizon Echospeed, General Electric), including T1 volume and dual-echo whole-brain $\mathrm{T} 2 \mathrm{map}^{16}$, which identified left hippocampal sclerosis and normal right hippocampus in all patients. Hippocampal volumetry (hippocampal volume corrected for total intracranial volume) was carried out according to a previously published protocol ${ }^{17}$. Maps of GMD were created using SPM99 software ${ }^{43}$ and the techniques of $\mathrm{VBM}^{21}$. In brief, the T1 volume for each subject was transformed into standard anatomical space and segmented into gray matter, white matter and CSF. The gray matter images were then smoothed with a 6-mm Gaussian kernel, to produce images of voxel-by-voxel GMD. The T2 map for each subject was also transformed into the standard anatomical space and was smoothed with a 6-mm Gaussian kernel.

Functional imaging. Subjects were scanned at 2T (Siemens VISION, Siemens), with continuous acquisition of gradient-echo echo-planar $\mathrm{T} 2{ }^{*}$-weighted image (EPI) volumes that provide blood oxygenation level-dependent contrast, with 33 slices covering the whole brain (repetition time 2.5 s). SPM99 was used for image analysis ${ }^{43}$. The first five EPI volumes of each subject were discarded. The remaining images were realigned and corrected for slice timing differences. A mean image was produced and volumetrically normalized to the SPM99 EPI template. All of the images from each subject were then transformed to the standard anatomical volume using these parameters and were smoothed with an 8-mm kernel.

Psychological task. During scanning, subjects were presented with 288 single words (one every $4.5 \mathrm{~s}$ ) previously used elsewhere ${ }^{44}$, including 36 emotionally aversive words ${ }^{7}$. Subjects indicated with a right-hand button press whether the word indicated a living or nonliving entity. A different set of stimuli from the same pool of 500 neutral words and 72 emotional words was used for each subject. Subjects carried out an unscanned surprise recognition memory test 90 min after scanning (Fig. 1). At recognition, single words were presented every $4.5 \mathrm{~s}$ in a manner identical to that used in scanning: 288 words presented during scanning, which were randomly mixed with 170 neutral foils and 36 emotional foils. For each stimulus, subjects were asked to indicate with a righthand button press whether they remembered seeing the word on the screen during scanning (R response), knew the word (K response) or did not know the word (new; $\mathrm{N}$ response) ${ }^{24}$. The encoding stimuli were conditionalized accordingly: correct $\mathrm{R}$ responses for neutral stimuli ( $\mathrm{Rn})$, correct $\mathrm{K}$ responses to neutral stimuli $(\mathrm{Kn})$, incorrect $\mathrm{N}$ responses to neutral stimuli $(\mathrm{Nn})$ and similarly for emotional stimuli ( $\mathrm{Re}, \mathrm{Ke}, \mathrm{Ne})$. A single category for all encoding task errors or missed responses was included; hence seven event types were indicated. To calculate recognition accuracy for each event type (Rn, Kn, Re, Ke), stimuli seen in the recognition test were classified as correct identifications ('hits') and incorrect responses to foils ('false alarms'). Recognition accuracy was calculated for each of these four event types as the hit rate minus the falsealarm rate. To calculate the recognition accuracy for $\mathrm{N}$ responses, stimuli in the recognition test were similarly classified as correct rejections and misses; recognition accuracy for $\mathrm{N}$ responses was calculated as the miss rate minus the correct rejection rate for neutral and emotional stimuli separately. The highest possible score is +1 , indicating perfect identification of stimuli present at encoding; the lowest score, -1 , indicates perfect identification of foils.

Imaging analysis—structural imaging data. Hippocampal volumes were entered into a regression with recognition accuracy scores. Voxel-by-voxel regression was also used to examine the relationships between GMD, T2 signal and recognition accuracy scores.

Imaging analysis-functional imaging data. To test for subsequent memory effects, imaging data were analyzed with a two-level random-effects analysis using an event-related design ${ }^{22}$. At the first level, trial-specific responses were modeled for each subject by convolving a $\delta$ function with the canonical hemodynamic response function to create regressors of interest. Seven regressors were entered for each subject, corresponding to that subject's responses (Rn, $\mathrm{Kn}, \mathrm{Nn}, \mathrm{Re}, \mathrm{Ke}, \mathrm{Ne}$, errors). Parameter estimates pertaining to the height of the hemodynamic response function for each regressor were calculated at each voxel. Contrasts of parameter estimates were calculated to produce four 'contrast images' for each patient ( $\mathrm{Rn}$ minus $\mathrm{Kn}$, Re minus Ke, Rn minus Nn, Re minus $\mathrm{Ne}$ ) and one contrast image for each normal subject (main effect of $\mathrm{R}$ minus K). These images were used for the second-level analyses.

At the second level, a one-sample $t$-test was used to examine the main effect of $\mathrm{R}$ minus $\mathrm{K}$ in the normal subjects. Regression was used to examine effects within the patients. We chose $P<0.05$, corrected for a small volume, as the threshold for significance. We report all MTL activations that survived this threshold and descriptively report MTL regions that showed uncorrected significance where this contributes to interpretation of the data. When examining the three-way interaction between left amygdala T2 and successful-encoding activity ( $\mathrm{R}$ minus $\mathrm{K}$ ) for emotional versus neutral items, we hypothesized that an effect would be seen in left hippocampus in the vicinity of the main effect of $\mathrm{R}$ minus $\mathrm{K}$ in normal subjects as reported previously ${ }^{26}$. Hence, we used a small volume comprising the cluster of voxels $\left(96 \mathrm{~mm}^{3}\right)$ below a threshold $P<0.05$ for the main effect of $\mathrm{R}$ minus $\mathrm{K}$ in left hippocampus in the normal subjects. Where we hypothesized an effect in amygdala, we used a small volume $\left(105 \mathrm{~mm}^{3}\right)$ in dorsal amygdala. Where we hypothesized an anterior MTL effect of uncertain location, we used a small volume including anterior hippocampus and amygdala $\left(2,400 \mathrm{~mm}^{3}\right)$.

Note: Supplementary information is available on the Nature Neuroscience website.

\section{ACKNOWLEDGMENTS}

We would like to thank the clinicians of the Department of Clinical and Experimental Epilepsy (J. Duncan, L. Sander, M. Walker, H. Cock, S. Sisodiya and M. Koepp) for referring patients to the study and P. Bartlett, Chief Radiographer at the Chalfont Centre for Epilepsy, for providing the volume and T2 data. M.P.R. is funded by a fellowship of the Medical Research Council, UK. R.J.D. is supported by a Wellcome Trust Programme Grant.

\section{COMPETING INTERESTS STATEMENT}

The authors declare that they have no competing financial interests.

Received 9 September; accepted 8 December 2003

Published online at http://www.nature.com/neuroscience/

1. Gallagher, M. \& Chiba, A.A. The amygdala and emotion. Curr. Opin. Neurobiol. 6, 221-227 (1996)

2. Cahill, L. \& McGaugh, J.L. Mechanisms of emotional arousal and lasting declarative memory. Trends Neurosci. 21, 294-299 (1998).

3. Dolan, R.J. Emotion, cognition and behavior. Science 298, 1191-1194 (2002)

4. Hamann, S.B., Ely, T.D., Grafton, S.T. \& Kilts, C.D. Amygdala activity related to enhanced memory for pleasant and aversive stimuli. Nat. Neurosci. 2, 289-293 (1999).

5. Phelps, E.A., LaBar, K.S. \& Spencer, D.D. Memory for emotional words following unilateral temporal lobectomy. Brain Cogn. 35, 85-109 (1997).

6. Adolphs, R., Cahill, L., Schul, R. \& Babinsky, R. Impaired declarative memory for emotional material following bilateral amygdala damage in humans. Learn. Mem. 4, 291-300 (1997).

7. Strange, B.A., Henson, R.N., Friston, K.J. \& Dolan, R.J. Brain mechanisms for detecting perceptual, semantic, and emotional deviance. Neuroimage 12, 425-433 (2000)

8. Cahill, L. et al. Amygdala activity at encoding correlated with long-term, free recall of emotional information. Proc. Natl. Acad. Sci. USA 93, 8016-8021 (1996).

9. McGaugh, J.L., Cahill, L. \& Roozendaal, B. Involvement of the amygdala in memory storage: interaction with other brain systems. Proc. Natl. Acad. Sci. USA 93, 13508-13514 (1996).

10. Squire, L.R. \& Zola-Morgan, S. The medial temporal lobe memory system. Science 253, 1380-1386 (1991).

11. Frisk, V. \& Milner, B. The role of the left hippocampal region in the acquisition and retention of story content. Neuropsychologia 28, 349-359 (1990).

12. Smith, M.L. \& Milner, B. The role of the right hippocampus in the recall of spatial location. Neuropsychologia 19, 781-793 (1981).

13. Alkire, M.T., Haier, R.J., Fallon, J.H. \& Cahill, L. Hippocampal, but not amygdala, activity at encoding correlates with long-term, free recall of nonemotional information. Proc. Natl. Acad. Sci. USA 95, 14506-14510 (1998).

14. Duncan, J.S. Imaging and epilepsy. Brain 120, 376-389 (1997).

15. Woermann, F.G. et al. Regional changes in hippocampal T2 relaxation and volume: a quantitative magnetic resonance imaging study of hippocampal sclerosis. J. Neurol. Neurosurg. Psychiatry 65, 656-664 (1998).

16. Duncan, J.S., Bartlett, P. \& Barker, G.J. Technique for measuring hippocampal T2 relaxation time. AJNR Am. J. Neuroradiol. 17, 1805-1810 (1996).

17. Van Paesschen, W. et al. Quantitative hippocampal MRI and intractable temporal lobe epilepsy. Neurology 45, 2233-2240 (1995). 
18. Bartlett, P.A., Richardson, M.P. \& Duncan, J.S. Measurement of amygdala T2 relax ation time in temporal lobe epilepsy. J. Neurol. Neurosurg. Psychiatry 73, 753-735 (2002).

19. Hudson, L.P. et al. Amygdaloid sclerosis in temporal lobe epilepsy. Ann. Neurol. 33 , 622-631 (1993).

20. Helmstaedter, C. \& Kurthen, M. Memory and epilepsy: characteristics, course, and influence of drugs and surgery. Curr. Opin. Neurol. 14, 211-216 (2001).

21. Ashburner, J. \& Friston, K.J. Voxel-based morphometry - the methods. Neuroimage 11, 805-821 (2000).

22. Friston, K.J. et al. Event-related fMRI: characterizing differential responses. Neuroimage 7, 30-40 (1998).

23. Craik, F.I.M. \& Lockhart, R.S. Levels of processing: A framework for memory. J. Verbal Learn. Verbal Behav. 11, 671-684 (1972).

24. Tulving, E. Memory and consciousness. Can. Psychol. 26, 1-12 (1985).

25. Windmann, S. \& Kutas, M. Electrophysiological correlates of emotion-induced recognition bias. J. Cogn. Neurosci. 13, 577-592 (2001).

26. Richardson, M.P., Strange, B.A., Duncan, J.S. \& Dolan, R.J. Preserved verbal memory function in left medial temporal pathology involves reorganisation of function to right medial temporal lobe. Neuroimage 20, S112-119 (2003).

27. Van Paesschen, W. et al. Quantitative neuropathology and quantitative magnetic resonance imaging of the hippocampus in temporal lobe epilepsy. Ann. Neurol. 42, 756-766 (1997).

28. Van Paesschen, W. et al. The spectrum of hippocampal sclerosis: a quantitative magnetic resonance imaging study. Ann. Neurol. 41, 41-51 (1997).

29. Kalviainen, R. et al. MRI-based hippocampal volumetry and T2 relaxometry: correlation to verbal memory performance in newly diagnosed epilepsy patients with leftsided temporal lobe focus. Neurology 48, 286-287 (1997).

30. Kilpatrick, C. et al. Degree of left hippocampal atrophy correlates with severity of neuropsychological deficits. Seizure 6, 213-218 (1997)

31. Lencz, T. et al. Quantitative magnetic resonance imaging in temporal lobe epilepsy: relationship to neuropathology and neuropsychological function. Ann. Neurol. 31, 629-637 (1992).

32. Briellmann, R.S., Kalnins, R.M., Berkovic, S.F. \& Jackson, G.D. Hippocampal pathology in refractory temporal lobe epilepsy: T2-weighted signal change reflects dentate gliosis. Neurology 58, 265-271 (2002).

33. Golby, A.J. et al. Memory lateralization in medial temporal lobe epilepsy assessed by functional MRI. Epilepsia 43, 855-863 (2002).

34. Dupont, S. et al. Episodic memory in left temporal lobe epilepsy: a functional MRI study. Brain 123, 1722-1732 (2000).

35. Bellgowan, P.S. et al. Side of seizure focus predicts left medial temporal lobe activation during verbal encoding. Neurology 51, 479-484 (1998).

36. Richter-Levin, G. \& Akirav, I. Amygdala-hippocampus dynamic interaction in relation to memory. Mol. Neurobiol. 22, 11-20 (2000).

37. Pitkanen, A., Pikkarainen, M., Nurminen, N. \& Ylinen, A. Reciprocal connections between the amygdala and the hippocampal formation, perirhinal cortex, and postrhinal cortex in rat. A review. Ann. NY Acad. Sci. 911, 369-391 (2000).

38. van Groen, T. \& Wyss, J.M. Extrinsic projections from area CA1 of the rat hippocampus: olfactory, cortical, subcortical, and bilateral hippocampal formation projections. J. Comp. Neurol. 302, 515-528 (1990).

39. Canteras, N.S. \& Swanson, L.W. Projections of the ventral subiculum to the amygdala, septum, and hypothalamus: a PHAL anterograde tract-tracing study in the rat. J. Comp. Neurol. 324, 180-194 (1992).

40. Krettek, J.E. \& Price, J.L. Projections from the amygdaloid complex and adjacent olfactory structures to the entorhinal cortex and to the subiculum in the rat and cat. J. Comp. Neurol. 172, 723-752 (1977).

41. Maren, S. \& Fanselow, M.S. Synaptic plasticity in the basolateral amygdala induced by hippocampal formation stimulation in vivo. J. Neurosci. 15, 7548-7564 (1995).

42. Seidenbecher, T., Laxmi, T.R., Stork, O. \& Pape, H.C. Amygdalar and hippocampal theta rhythm synchronization during fear memory retrieval. Science 301, 846-850 (2003).

43. Friston, K.J. et al. Statistical parametric maps in functional imaging: A general linear approach. Hum. Brain. Map. 2, 189-210 (1995).

44. Otten, L.J., Henson, R.N. \& Rugg, M.D. Depth of processing effects on neural correlates of memory encoding: relationship between findings from across- and withintask comparisons. Brain 124, 399-412 (2001). 